\title{
Current Insights into Research on Rice stripe virus
}

\author{
Won Kyong Cho ${ }^{\dagger, 1}$, Sen Lian ${ }^{\dagger, 1}$, Sang-Min Kim ${ }^{\dagger, 1,2}$, Sang-Ho Park ${ }^{1}$ and Kook-Hyung Kim ${ }^{1 *}$ \\ ${ }^{I}$ Department of Agricultural Biotechnology, Plant Genomics and Breeding Institute, Institute for Agriculture and Life Sciences, \\ College of Agriculture and Life Sciences, Seoul National University, Seoul 151-921, Korea \\ ${ }^{2}$ National Institute of Crop Science, Rural Development Administration, Suwon 441-707, Korea \\ (Received on October 28, 2012; Revised on November 27, 2012; Accepted on November 28, 2012)
}

Rice stripe virus (RSV) is one of the most destructive viruses of rice, and greatly reduces rice production in China, Japan, and Korea, where mostly japonica cultivars of rice are grown. $\mathrm{RSV}$ is transmitted by the small brown plant-hopper (SBPH) in a persistent and circulative-propagative manner. Several methods have been developed for detection of $\mathrm{RSV}$, which is composed of four single-stranded RNAs that encode seven proteins. Genome sequence data and comparative phylogenetic analysis have been used to identify the origin and diversity of RSV isolates. Several rice varieties resistant to RSV have been selected and QTL analysis and fine mapping have been intensively performed to map RSV resistance loci or genes. RSV genes have been used to generate several genetically modified transgenic rice plants with RSV resistance. Recently, genome-wide transcriptome analyses and deep sequencing have been used to identify mRNAs and small RNAs involved in RSV infection; several rice host factors that interact with RSV proteins have also been identified. In this article, we review the current statues of RSV research and propose integrated approaches for the study of interactions among RSV, rice, and the SBPH.

Keywords : genome, quantitative trait locus, resistance, rice, Rice stripe virus

\section{Introduction to Rice stripe virus}

Rice (Oryza sativa) is one of the most important crops in the world and is a main food in Asian countries including China, India, Indonesia, Japan, Korea, and the Philippines (Wang et al., 2005). Rice stripe virus (RSV) causes dramatic losses in rice production (Hibino, 1996). RSV is the member of genus Tenuivirus, which has not yet been assigned to a virus family. RSV mainly infects rice plants

\footnotetext{
These authors contributed equally to this work.

*Corresponding author.

Phone) +82-2-880-4677, FAX) +82-2-873-2317

E-mail)kookkim@snu.ac.kr
}

but also infects maize, wheat, oat, foxtail millet, and several weeds (Falk and Tsai, 1998; Lian et al., 2011). RSV can also infect Arabidopsis, which is widely used as a model plant (Sun et al., 2011). Over the past several decades, RSV has been frequently reported in China, Japan, and Korea. In Korea, RSV was previously reported only from southern areas but has been recently observed over a large range of altitudes, suggesting that the virus is rapidly spreading (Lee et al., 2008). In China, RSV has recently reemerged in Zhejiang province, eastern China, and the spread of RSV has been associated with climate change (Wang et al., 2008); this study demonstrated that RSV frequently infected the earliest sown seedlings and that young seedlings tend to be more susceptible than older plants (Wang et al., 2008). Since 2000, RSV has appeared in the Yangtze River region and generally caused a $30-40 \%$ reduction in yield in eastern China (Wu et al., 2009). The occurrence of RSV in western Japan in 2008 was caused by viruliferous planthoppers that migrated from China (Otuka et al., 2010). Interestingly, RSV has been reported only in China, Japan, Korea, and Taiwan, where japonica cultivars, which are susceptible to RSV, are usually grown (Abo and Sy, 1997).

RSV is transmitted by the small brown plant-hopper (SBPH) (Laodelphax striatellus Fallén) in a persistent and circulative-propagative manner (Falk and Tsai, 1998). However, the ability to acquire RSV is highly variable among SBPH isolates (Kisimoto, 1967). Specifically, RSV could be acquired from frozen infected leaves and then transmitted by the SBPH in the laboratory condition (Zhang et al., 2007).

\section{Identification and detection of RSV in rice plants and SBPH vectors}

Disease symptoms in rice plants infected by RSV include chlorosis, weakness, necrosis, and stunted growth (Satoh et al., 2010). Several methods have been developed to detect RSV in rice plants or SBPH vectors, and these include enzyme-linked immunosorbent assay (ELISA), western blot analysis, reverse transcription-polymerase chain reaction 
(RT-PCR), and RT loop-mediated isothermal amplification (LAMP). ELISA, which has been widely used for detection of RSV in high-throughput experiments (Takahashi et al., 1991), required the development of several antibodies specific to RSV proteins (Lian et al., 2011; Liang et al., 2005; Suzuki et al., 1992). Such antibodies can be generated as recombinant proteins or as synthetic polypeptides (Lian et al., 2011). The developed antibodies have also been used to localize specific RSV proteins in rice plants and SBPH in vivo (Liang et al., 2005; Suzuki et al., 1992). Western blot analysis with six antibodies detected all six RSV proteins in RSV-infected plants (Liang et al., 2005). A limitation of both ELISA and western blot analysis is the time and cost required for antibody production. Recently, RT-PCR has being widely used to detect RSV (Cai et al., 2003; Zhang et al., 2008). Although RT-PCR is very specific and sensitive, it requires expensive equipment and long amplification times. Immuno- and virion-capture RTPCR have been developed, and these have the advantage of not requiring the extraction of RNA from samples (Lian et al., 2011; Liang et al., 2005; Suzuki et al., 1992). Recently, a LAMP approach was developed to detect nine viruses including RSV in rice (Le et al., 2010). LAMP, which does not require thermo-cycling, is specific, fast, accurate, and simple (Notomi et al., 2000).

\section{Organization of the RSV genome}

The RSV genome is composed of four single-stranded RNAs that encode seven proteins (Fig. 1). The morphological characteristics of RSV RNAs were examined after the separation of RNAs by sucrose density gradient centrifugation (Ishikawa et al., 1989). Each of the four particle contains circular filaments, and the average length of each particle containing RNA1-4 is 510, 610, 840, and 2,110 nm, respectively (Ishikawa et al., 1989). RNA1 is the largest RNA segment and is a negative-sense RNA. A single ORF in the viral-complementary sense RNA1 (vc RNA1) encodes a 337-kDa protein referred to as the RNA-dependent RNA polymerase (RdRp) (Barbier et al., 1992; Toriyama et al., 1994). Based on the RdRp sequence, RSV is regarded as a member of the genus Tenuivirus (Toriyama et al., 1994). The other three RSV RNAs (RNA2, RNA3, and RNA4) are ambisense in their coding strategy (Hamamatsu et al., 1993). The complete nucleotide sequences for RNA2, RNA3, and RNA4 indicate that each RNA segment encodes two proteins (Takahashi et al., 1993; Zhu et al., 1991; Zhu et al., 1992). RNA2 encodes P2 (silencing suppressor; 22.8 $\mathrm{kDa}$ ) from the viral-sense RNA2 (vRNA2) and PC2 (glycoprotein; $94 \mathrm{kDa}$ ) from the vcRNA2 (Takahashi et al., 1993). The P3 (nonstructural protein, NS3; $23.9 \mathrm{kDa}$ ) and nucleocapsid protein $(\mathrm{CP} ; 35 \mathrm{kDa})$ are produced from

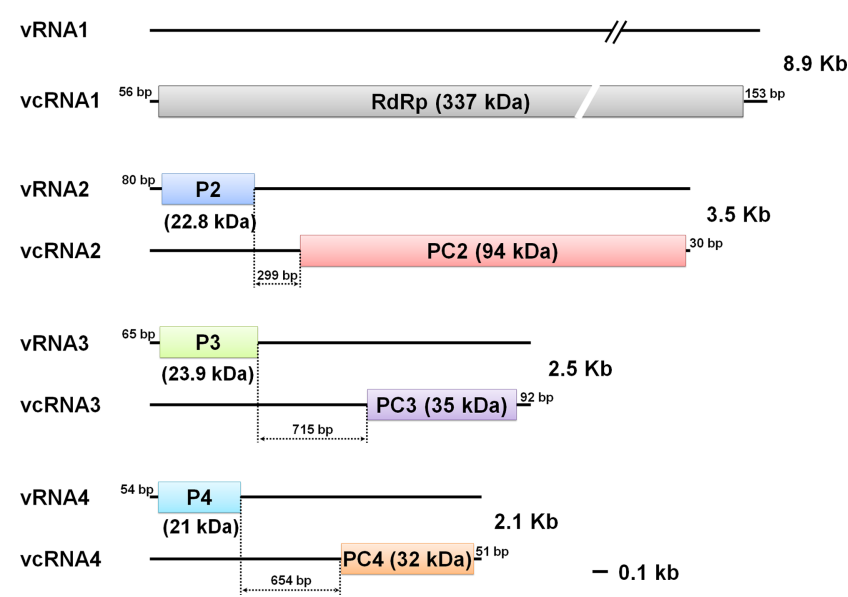

Fig. 1. Organization of the RSV genome. RSV consists of four RNAs: RNA1, RNA2, RNA3, and RNA4. Viral-sense RNAs and viral-complementary sense RNAs are abbreviated vRNA and vcRNA, respectively, with the corresponding RNA number. The black line indicates the length of nucleotides for each RNA, and the colored rectangular represents the corresponding ORF with the predicted protein size. The sizes (in bp) of 5', 3', and intergenic regions are also indicated. The name of each protein is as follows: RdRp in RNA1; P2 (silencing suppressor) and PC2 (glycoprotein) in RNA2; and P3 (nonstructural protein, NS3), PC3 (nucleocapsid protein, CP) in RNA3; P4 (nonstructural disease-specific protein, SP), and PC4 (movement protein, MP) in RNA4.

vRNA3 and vcRNA3, respectively (Hayano et al., 1990; Kakutani et al., 1991). A nonstructural disease-specific protein (SP; $20.5 \mathrm{kDa}$ ) and PC4 (movement protein, MP; $32 \mathrm{kDa}$ ) are encoded by vRNA4 and vcRNA4, respectively (Hayano et al., 1990; Kakutani et al., 1990). Interestingly, 5' and 3 ' terminal sequences of RSV are complementary to each other and might form a panhandle structure (Takahashi et al., 1990).

A previous study that examined the nucleotide sequences of the 5' termini of RNA3 and RNA4 found that the mRNAs from both viral and viral complementary sequences possess from 10 to 23 non-viral sequences that might have originated from host cellular mRNAs and that are thought to ensure efficient translation of viral mRNA (Shimizu et al., 1996). Coinfection with RSV and Cucumber mosaic virus (CMV) in Nicotiana benthamiana indicated that CMV RNAs served as cap donors for the initiation of RSV transcription (Yao et al., 2012). The 5' end of the cleaved CMV RNAs was complementary to the $3^{\prime}$ end of the RSV genome sequences. Repetitive priming and realignment evidently converted short CMV cap donors into longer sizes, which could be useful for long-distance elongation of RSV (Yao et al., 2012). This "cap-snatching mechanism" has been well characterized in hantavirus, influenza virus, and other viruses (Cheng and Mir, 2012; Dias et al., 2009). 


\section{Genetic diversity of RSV isolated from different geo- graphical regions}

Complete genome sequences of various RSV isolates are currently available. A total of $13 \mathrm{RSV}$ isolates from Korea were used for complete genome sequencing (Jonson et al., 2009); significant differences were detected in the intergenic regions of each RSV RNA, and comparative phylogenetic analysis with other isolates from China and Japan suggested that RSV isolates in Korea may have originated from distinctive ancestors by either reassortment or recombination events. To elucidate the genetic diversity and population structure of RSV isolates in China, nucleotide sequences were determined for a large number of RSV isolates collected from 1997-2004 (Wei et al., 2009). The RSV isolates in China could be divided into three subtypes. The populations from eastern China were composed only of subtype I/IB isolates, while the populations from Yunnan province (southwest China) contained mostly subtype II isolates. The RSV isolates from two different districts in China displayed low genetic diversity, indicating substantial gene flow (Wei et al., 2009). The full-genome sequence of RSV isolate 'Zhejiang' revealed that RdRp contains a domain referred to as ovarian tumour (OTU), and indicated that RdRp might produce two different proteins; a novel inverted repeat motif also was detected within the intergenic regions of the ambisense RNAs (Zhang et al., 2007). Both Zhang et al. (2007a) and a previous study (Wei et al., 2003) that analyzed the intergenic region of RNA4 for 22 isolates in China suggested the possible role of the intergenic region in transcription termination. Using available RdRp amino acid sequences, we constructed a phylogenetic tree in which RSV isolates were divided into two groups (Fig. 2). The first group includes several RSV isolates from Korea, China, and Japan while the second group possesses seven RSV isolates only from China. These results suggested the RSV isolates in the first group might be diverged from the same RSV ancestor.

\section{Functions of RSV proteins}

A recent study confirmed that $\mathrm{PC} 2$ tagged with enhanced GFP was cleaved into two proteins, PC2-N and PC2-C (Zhao et al., 2012). Both cleaved PC2 proteins are independently targeted to the endoplasmic reticulum (ER), indicating that PC2 seems to contain three domains for ER targeting (Zhao et al., 2012). Another study showed that PC2 protein, which is similar to membrane glycoproteins of viruses in the family Bunyaviridae, could be expressed on the surface of insect Sf9 cells (Zhao et al., 2012).

The $\mathrm{P} 3$ protein, which can be expressed in the rice host as well as in the SBPH vector, forms inclusion bodies in both

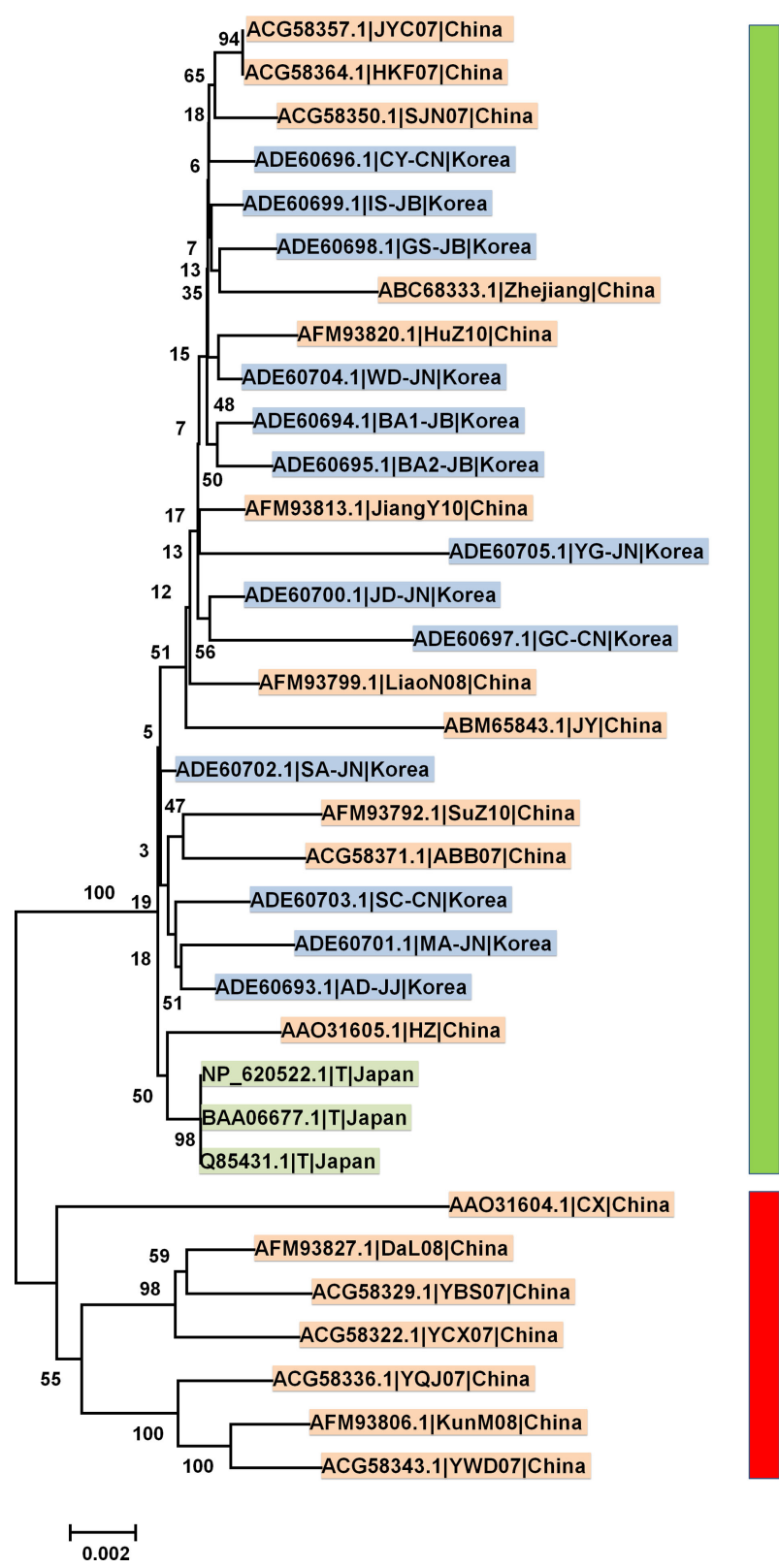

Fig. 2. The phylogenetic tree of $34 \mathrm{RSV}$ isolates from China, Japan, and Korea. A total of $34 \mathrm{RdRp}$ amino acid sequences for the $34 \mathrm{RSV}$ isolates were retrieved. The phylogenetic tree was constructed based on the neighbor-joining method with 1000 bootstraps using MEGA5 program (www.megasoftware.net). Numbers at each node indicate bootstrap values. The scale bar represents 0.1 substitutions per amino acid site. Protein accession number, name, and country for each isolate are indicated. Orange, green, and blue colors indicate isolates from China, Japan, and Korea, respectively. The phylogenetic tree reveals two groups of RSV isolates. The first group (indicated by the green bar) is composed of isolates from the three countries while the second group (indicated by the red bar) contains isolates only from China.

plant and insect cells (Takahashi et al., 2003). P3 protein was identified as a RNA-silencing suppressor and can 
reduce the levels of small interfering RNAs (siRNAs) by binding to those small RNAs but not to double-stranded RNAs (Xiong et al., 2009). P3 tagged with green fluorescent protein (GFP) was targeted to the nucleus, and both $\mathrm{N}$ - and C-terminal regions of $\mathrm{P} 3$ are important for inhibition of RNA silencing (Xiong et al., 2009). In contrast, a recent study with dsRNA-binding mutants and quantitative biochemical approaches revealed that dimeric P3 is a sizeindependent dsRNA-binding protein that can bind to long dsRNA with two or more copies (Shen et al., 2010). This study provided evidence that the RNA-silencing activity of P3 is correlated with its dsRNA-binding activity (Shen et al., 2010). A previous study that measured the nucleic acidbinding activity of six RSV proteins suggested that three viral proteins (P3, PC3, and PC4) could bind to single- and double-stranded RNA3 and RNA4 of RSV (Liang et al., 2005).

A movement-deficient mutant of Potato virus X (PVX) and biolistic bombardment were recently used to demonstrate that PC4 of RSV is a viral-movement protein (Xiong et al., 2008). An Agrobacterium-mediated transient assay with enhanced green fluorescent fusion protein and immune cytochemistry suggested plasmodesmal localization of PC4 (Xiong et al., 2008). In addition, a movement-deficient mutant of Tobacco mosaic virus (TMV) and mutagenesis were used to identify the regions of PC4 required for cellto-cell movement, long-distance movement, and leaf necrosis response (Zhang et al., 2012). Moreover, PC4 was determined to target plasmodesmata via the ER to Golgi secretory pathway and by using the actin-myosin VIII-1 motility system (Yuan et al., 2011).

\section{Identification of quantitative trait loci (QTL) for RSV resistance}

For control of the rice stripe disease, several RSV-resistant

Table 1. Identified QTLs that confer resistance to RSV in rice

\begin{tabular}{|c|c|c|c|c|c|c|}
\hline Cultivar & No. of QTL & Resistance gene & Markers and location & Fine mapping & $\begin{array}{l}\text { No. of } \\
\text { genes }\end{array}$ & Publication \\
\hline 'Modan' & 1 QTL (Chr11) & $S t v b-i$ & & & & Saito et al., 1998 \\
\hline 'Kanto 72' & $\begin{array}{l}2 \text { QTLs (Chr2 } \\
\text { and Chr11) }\end{array}$ & & & & & $\begin{array}{l}\text { Maeda et al., } \\
2004\end{array}$ \\
\hline $\begin{array}{l}\text { A cross of 'Kinmaze' } \\
\text { (japonica) and } \\
\text { 'DV85' (indica) }\end{array}$ & $\begin{array}{l}3 \text { QTLs (Chr1, } \\
\text { Chr7, and Chr11) }\end{array}$ & $\begin{array}{c}q S t v 1 \text { ('Kinmaze'), } \\
q S t v 7 \text { and } \\
q S t v 11 \text { ('DV85') }\end{array}$ & $\begin{array}{c}q S t v 11(\mathrm{XNpb} 202 \\
\text { and } \mathrm{C} 1172)\end{array}$ & $q S t v 11(27.5 \mathrm{cM})$ & & Ding et al., 2004 \\
\hline 'Dular' & $\begin{array}{l}1 \text { QTL (Chr3) and } \\
3 \text { QTLs (Chr11) }\end{array}$ & $\begin{array}{l}q S T V 3(\mathrm{Chr} 3), \\
\text { STV-11a (Chr11), } \\
\text { STV-11b (Chr11), } \\
\text { STV-11c (Chr11) }\end{array}$ & $\begin{array}{c}q S T V 3(\mathrm{RM} 7324- \\
\mathrm{RM} 3586), q S T V-11 a \\
\text { (RM1124-SSR20), qSTV- } \\
11 b(\mathrm{RM} 287-\mathrm{RM} 209), \\
q S T V-11 c(\mathrm{RM} 209-\mathrm{RM} 21)\end{array}$ & & & Wu et al., 2008 \\
\hline $\begin{array}{c}\text { A cross of } \\
\text { 'Zhaiyeqing8' } \\
\text { and 'Jingxi17' }\end{array}$ & $\begin{array}{l}1 \text { QTL (Chr1) and } \\
1 \text { QTL (Chr11) }\end{array}$ & $\begin{array}{c}q S B P H 1 \text { and } \\
q S B P H 11\end{array}$ & & & & $\begin{array}{l}\text { Zhang et al., } \\
2010\end{array}$ \\
\hline '502' & 1 QTL (Chr11) & RSV1 & RM457 and SR1 & $\begin{array}{l}\text { Between } 4.5 \mathrm{cM} \\
\text { and } 2.9 \mathrm{cM}\end{array}$ & & Zhao et al., 2010 \\
\hline 'Teqing' & 1 QTL (Chr11) & qSTV11TQ & CAPs1 and Indel 4 & $q S T V 11 T Q(55.7 \mathrm{~kb})$ & 9 & Wu et al., 2011 \\
\hline 'Zhendao 88' & 1 QTL (Chr11) & & RM229 and OPO11 & $4.7 \mathrm{cM}$ & & Zhou et al., 2011 \\
\hline 'IR24' (indica) & $\begin{array}{l}3 \text { QTLs (Chr3, } \\
\text { Chr7, and Chr11) }\end{array}$ & $q S T V 3, q S T V 11-i$ & $\begin{array}{c}q S T V 3 \text { (RM5748- } \\
\text { RM14975) and } \\
q S T V 11-i(\mathrm{R} 45-\mathrm{R} 48)\end{array}$ & $\begin{array}{c}q S T V 11-i \\
\text { (73.6 kb region) }\end{array}$ & 11 & $\begin{array}{l}\text { Wang et al., } \\
2011\end{array}$ \\
\hline 'Kasalath' & $\begin{array}{c}2 \text { QTLs (Chr7 } \\
\text { and Chr11) }\end{array}$ & $\begin{array}{c}q S T V 7, \\
q S T V 11 K A S\end{array}$ & $\begin{array}{c}q S T V 11 K A S \\
(\mathrm{C} 1 \text { and } \mathrm{R} 53)\end{array}$ & $\begin{array}{c}q S T V 11 K A S \\
\text { (39.2 kb region) }\end{array}$ & 7 & $\begin{array}{l}\text { Zhang et al., } \\
2011\end{array}$ \\
\hline $\begin{array}{l}\text { 'Habataki' } \\
\text { (indica) }\end{array}$ & $\begin{array}{l}1 \text { QTL (Chr3) and } \\
2 \text { QTLs (Chr11) }\end{array}$ & $\begin{array}{c}q S T V 11 H A B-1 \text { and } \\
q S T V 11 H A B-2 \\
(\mathrm{Chr} 11)\end{array}$ & & $\begin{array}{c}q S T V 11 H A B-1 \\
(333.2 \mathrm{~kb}) \text { and } \\
q S T V 11 H A B-2 \\
(203.9 \mathrm{~kb})\end{array}$ & & $\begin{array}{l}\text { Zhang et al., } \\
2012\end{array}$ \\
\hline $\begin{array}{l}\text { A cross of 'Shin- } \\
\text { gwang' and 'Ilpum' }\end{array}$ & 1 QTL (Chr11) & $q S T V 11 S G$ & G257 and S2260 & $150 \mathrm{~kb}$ & 21 & $\begin{array}{l}\text { Kwon et al., } \\
2012\end{array}$ \\
\hline
\end{tabular}


varieties have been selected for resistance breeding. The first reported RSV-resistance loci from Japanese upland rice varieties were $S t v-a$ (located on chromosome 6) and $S t v-b$ (located on chromosome 11) (Washio et al., 1968). Because the $S t v-b$ locus provides incomplete resistance to RSV and the $S t v b-i$ from indica rice cultivar 'Modan' provided complete resistance to RSV, $S t v b-i$ was introduced to various japonica cultivars in spite of its incomplete dominance (Washio et al., 1968; Pan et al., 2005; Kwon et al., 2012).

After the development of molecular genetics and polymorphic molecular markers (McCough and Doerge, 1995), QTL analysis and fine mapping have often been used to map RSV resistance loci (Table 1). A cross of susceptible japonica and resistant cultivars, generally indica rice cultivars, has been widely used. The Stvb-i from rice 'Modan' was mapped at $35.85 \mathrm{cM}$ on chromosome 11 (HayanoSaito et al., 1998). Fine mapping determined that $S t v b-i$ is located within a $286-\mathrm{kb}$ region that is covered by two overlapped BAC clones on chromosome 11 (Hayano-Saito et al., 2000).

QTL analyses using molecular markers can be used to identify the integressed chromosome regions in various resistant varieties. As a result, many QTLs related to RSV resistance have been detected. The indica rice 'Dular' carries four QTLs responsible for RSV resistance, and these include $q S T V 3$ on chromosome 3 and three QTLs ( $q S T V$ $11 a, q S T V-11 b$, and $q S T V-11 c$ ) on chromosome 11 (Wu et al., 2009). A study that used 85 backcross inbred lines of Sasanishiki (japonica)/Habataki (indica) identified three QTLs; one on chromosome 3 and two (qSTV11 ${ }^{\text {HAB }}-1$ and qSTV11 ${ }^{H A B}$-2) on chromosome 11 (Zhang et al., 2012). In addition, recombinant inbred lines from a cross of 'Kinmaze' (japonica)/'DV85' (indica) were used to find QTLs responsible for resistance to RSV (Ding et al., 2004); three QTLs on chromosome 1, 7, and 11 were identified. 'DV85' allele was associated with increased resistance at $q S T v 7$ and qSTv11 while 'Kinmaze' allele was associated with RSV resistance at $q S T v 1$. Moreover, using the cross of ' 502 ' (resistant to RSV) and '5112' (susceptible to RSV), RSV1 gene on chromosome 11 was mapped by RM457 and SR1 markers (Zhao et al., 2010). Another Japanese upland rice, 'Kanto 72', contains one QTL on chromosome 2 and one on chromosome 11 (Maeda et al., 2006); the QTL on chromosome 11 seemed to reduce the infection rate while the QTL on chromosome 2 reduced disease symptoms. The rice cultivar 'BL 1', which was originally identified as an elite germplasm that is resistant to the rice blast fungus, is also resistant to RSV; BL 1 resistance to RSV is independent of Pib and Stvb-i (Ise et al., 2002).

Recently, fine mapping along with rice genome sequence data have been used to identify potential candidate genes that provide resistance to RSV (Kwon et al., 2012; Wang et al., 2011; Wu et al., 2011; Zhang et al., 2011). For example, fine mapping of the indica cultivar 'Teqing', which has a high level of resistance to RSV, was used to identify a major resistance gene referred to as $q S T V 11^{T Q}$ (Wu et al., 2011). To map finely resistance loci for 'Zhendao 88' and 'Teqing' cultivars, RM209 and RM229 markers were commonly used and this study suggested that $q S T V 11^{T Q}$ and $S t v b-i$ might be allelic (Wu et al., 2011). $q S T V 11^{T Q}$ was localized to a $55.7-\mathrm{kb}$ region that contained nine genes including four genes associated with transposons, two genes encoding ATP-binding proteins, and a gene encoding brassinosteroid insensitive 1-associated receptor-like kinase 1 (Wu et al., 2011). In a study with rice indica cultivar 'IR24', which contains positive alleles at three loci, backcross analysis detected $q S T V 3$ and $q S T V 11-i$, and an allelic test confirmed that $q S T V 11-i$ is not allelic with Stvb-i (Wang et al., 2011). Fine mapping in the latter study uncovered a region containing 11 rice genes for RSV resistance. Using 98 backcross inbred lines and artificial inoculation in greenhouse tests, researchers identified two QTLs ( $q S T V 7$ located on chromosome 7 and $q S T V 11^{K A S}$ located on chromosome 11) that are related to RSV resistance (Zhang et al., 2011). Fine mapping revealed that $q S T V 11^{K A S}$ was localized to a $39.2-\mathrm{kb}$ region that included seven genes. Among them, a gene encoding a sulfotransferase domain-containing protein (LOC_Os11g30910) could be responsible for RSV resistance. Another resistance gene, $q S T V 11^{S G}$ on chromosome 11, was also finely mapped (Kwon et al., 2012). With the use of InDel 11 and InDel 5 as markers, $q S T V 11^{S G}$ was localized to a 150-kb region containing 21 genes. Among them, three genes (LOC_Os11g31430, LOC_Os11g31450, and LOC Os11g31470) were strongly expressed in a susceptible variety (Kwon et al., 2012). The identification of resistance genes and marker-assisted selection-based breeding will clearly be important for the development of RSV-resistant rice cultivars with excellent taste (Pan et al., 2009).

\section{Generation of transgenic rice plants resistant to RSV}

Because most cultivated japonica rice plants contain the same resistant locus, the possibility of resistance breakdown is very high (Wu et al., 2009). QTL analyses suggested that the major QTLs related to RSV resistance are clustered in a narrow region on chromosome 11. Thus, introgression to other cultivars by recombination might be difficult. Moreover, the QTLs located on other chromosomes (like chromosomes 1,2, and 7) confer only minor resistance (Ding et al., 2004; Maeda et al., 2006). Therefore, alternative approaches are needed to develop rice plants with resistance to RSV. One approach might be to generate transgenic rice plants (Table 2). The first transgenic rice plants, which were generated by electroporation of protoplasts, overexpressed 
Table 2. Transgenic rice plants with modified RSV resistance

\begin{tabular}{cccc}
\hline \hline Method & Gene & Resistance & Publication \\
\hline RNAi & PC2 (glycoprotein), PC3 (CP), & Resistant to RSV (PC3 and PC4) & Shimizu et al., 2011 \\
(Inverted Repeat) & P4 (SP), and PC4 (MP) & Not resistant to RSV (PC2 and P4) & Zhou et al., 2012 \\
RNAi & PC3 (CP) and P4 (SP) & Resistant to RSV & Jiang et al., 2012 \\
Antisense & OsRDR6 (Rice) & $\begin{array}{c}\text { Increased RSV infection and reduced } \\
\text { level of siRNA from RSV }\end{array}$ & Park et al., 2012 \\
RNAi & PC3 (CP) & Resistant to RSV & Ma et al., 2011 \\
RNAi & PC3 (CP), P4 (SP), and PC3/P4 & Chimeric PC3/P4 construct was the \\
most effective for RSV resistance & Zhang et al., 2012 \\
hpRNA & PC3 (CP), Three different promoters; & & Hayakawa et al., 1992 \\
Overexpression & CaMV 35S, Ubi, and PNZIP & & RC3 (CP)
\end{tabular}

the RSV CP gene (Hayakawa et al., 1992); the transgenic rice plants expressed high levels of $C P$ transcript and showed strong resistance to RSV infection. Since then, many CP-mediated transgenic rice plants have been developed (Ma et al., 2011; Park et al., 2012; Shimizu et al., 2011; Zhang et al., 2012; Zhou et al., 2012). RNA interference (RNAi) technology has also been frequently used to generate transgenic rice plants. For example, a recent study demonstrated that the double-stranded (ds) RNA-mediated RNAi system carrying the full length of the $C P$ gene generated transgenic rice plants resistant to RSV; these plants simultaneously suppressed transcription of the RSV genes $M P$ and NS3 (Park et al., 2012). In addition, three RNAi vectors with $\mathrm{CP}, \mathrm{SP}$, or chimeric $\mathrm{CP} / \mathrm{SP}$ gene sequences were used to make transgenic rice plants (Ma et al., 2011). RSV resistance was stronger for plants carrying the chimeric CP/SP RNAi than for plants carrying CP or SP RNAi. Similarly, an RNAi construct carrying CP and SP sequences of RSV was used to generate transgenic rice plants of the japonica varieties Suyunuo and Guanglingxiangjing (Zhou et al., 2012); the generated transgenic rice plants showed strong suppression of $S P$ and $C P$ transcripts and no obvious morphological and developmental defects. All seven RSV genes were used to generate transgenic rice plants that expressed inverted-repeat (IR) constructs (Shimizu et al., 2011). The transgenic plants carrying IR constructs specific for CP and MP were resistant to RSV, but plants carrying IR constructs specific for glycoprotein and non-structural protein were not.

Three promoters (Cauliflower mosaic virus 35S, polyubiqutin gene of maize, and Pharbitis nil leucine zipper gene) that differ in their tissue-specific activity were used to construct hairpin RNA expression vectors with an inverted repeat fragment of the RSV CP gene (Zhang et al., 2012); northern blot and GUS (beta-glucuronidase) assays showed that virus resistance was highly correlated with promoter activity. To demonstrate involvement of rice $R D R 6$ gene in host defense against RSV, researchers generated transgenic rice plants by inducing antisense RNA of OsRDR6 (Jiang et al., 2012); the OsRDR6AS plants exhibited a high level of $\mathrm{RSV}$ infection and a reduction in RSV-derived siRNA.

\section{Gene expression analysis in response to RSV infec- tion}

Gene expression analysis has been used to monitor molecular changes of host and virus during infection. When the expression patterns of RSV genes in RSV-infected rice and in viruliferous SBPH were assessed by quantitative realtime (RT) PCR, expression was highest in both rice and SPBH for the NS3 gene while expression of the SP gene was high only in rice (Li et al., 2012). The transcript level of RdRp was generally lower than those of other RSV genes. In another study, transcripts for defense genes like PRla, PAL, and CHS as well as for genes that encode diverse antioxidant enzymes were highly accumulated in an RSV resistant rice variety upon infection with RSV, suggesting the involvement of these genes in RSV resistance (Hao et al., 2011). A previous report showed that RSV infection induced expression of yuccal and an increase in endogenous indole acetic acid in the host, indicating that auxin is involved in RSV replication (Yang et al., 2010).

With the rapid development of the technology required for gene expression analysis, several genome-wide transcriptome analyses of rice in response to RSV infection have been performed. In one case, a microarray containing 43,494 oligo probes representing 59,000 rice gene loci was used to examine transcriptional changes of rice infected by RSV (Satoh et al., 2010). Samples were harvested at 3, 6, 9, and 12 days after infection. RSV infection led to the accumulation of transcripts for protein synthesis and energy production while genes for chloroplast and cell-structure components were down-regulated. Two previous studies used $44 \mathrm{~K}$ and $22 \mathrm{~K}$ microarrays to identify rice $\mathrm{AP} 2 /$ 
EREBP and NAC transcription factor genes that are regulated by RSV infection (Nuruzzaman et al., 2010; Sharoni et al., 2011). These studies demonstrated that RSV caused more severe disease symptoms than Rice tungro spherical virus (RTSV) and Rice dwarf virus (RDV) (Nuruzzaman et al., 2010; Sharoni et al., 2011). Recently, a systemic gene analysis method was developed to identify putative rice factors responsible for resistance to RSV. Rice plants of two lines (RSV-resistant NIL22 and RSV-susceptible NIL37) that were RSV-infected or uninfected were used for gene expression profiling using a rice oligo microarray (Kim et al., 2012). Among 237 genes showing differential expression between the two lines, 11 genes were selected based on known QTL. Among 417 genes showing differential expression between RSV-infected and uninfected plants, 21 genes related to RSV resistance were confirmed by RT-PCR and a network-based method. This study demonstrates that the combining of gene expression profiles and known QTL information can facilitate the finding of rice genes associated with RSV resistance (Kim et al., 2012).

Deep sequencing-based approaches are ideal for revealing small RNA populations derived from the host or virus. A recent deep sequencing study revealed the viral siRNAs derived from RSV in RSV-infected rice plants (Yan et al., 2010). The identified siRNAs ranged from 20 to $22 \mathrm{bp}$, and similar amounts of siRNAs were identified from sense and antisense strands of RSV. Most of the siRNAs were derived from coding sequences as well as from the 5 ' regions of the RSV RNAs. Compared to the RSV genome, RSV siRNAs exhibited a higher $\mathrm{G}$ and lower $\mathrm{C}$ content (Yan et al., 2010). In another study, rice miRNAs in rice infected by RSV were identified by small RNA deep sequencing (Du et al., 2011). This study showed that RSV infection induced expression of some rice miRNAs but not their corresponding miRNAs. RSV infection also increased expression of OsDCLs and OsAGOs. Gaining detailed insight into the rice transcriptome now requires that researchers integrate all transcriptome data from microarrays, small RNAs from $\mathrm{RSV}$, and rice miRNAs.

\section{Identification of rice host factors interacting with RSV proteins}

RSV uses host factors to complete its life cycle, and it is therefore important to identify host factors that interact with RSV proteins or RNAs. However, only three rice host factors interacting with RSV proteins have been identified. Based on yeast two-hybrid ( $\mathrm{Y} 2 \mathrm{H})$ systems, two rice chaperone proteins including a type I DnaJ and a small Hsp were identified as host factors interacting with RSV MP, and their specific interaction was confirmed by coimmunoprecipitation (Lu et al., 2009). Y2H and biomolecular fluore- scence complementation (BiFC) experiments demonstrated that another rice host protein, which is homologous to Arabidopsis suppressor of gene silencing (AtSGS3), can bind to RSV P2 (Du et al., 2011). The expression of the RSV P2 gene enhanced infectivity and pathogenicity of Potato virus $X$ in $N$. benthamiana, confirming the functional role of $\mathrm{P} 2$ as a silencing suppressor. A recent study identified five SBPH host proteins (a receptor for activated protein kinase $\mathrm{C}$ (RACK), glyceraldehyde-3-phosphate dehydrogenase (GAPDH3), and three ribosomal proteins) that interact with RSV proteins (Li et al., 2011). A dot immunobinding assay indicated that all five proteins interacted with RSV particles; a Y2H experiment indicated, however, that RACK and GAPDH3 did not bind to RSV CP protein. RACK and GAPDH3 might be involved in epithelial transcytosis, and the three ribosomal proteins might be involved in RSV replication (Li et al., 2011).

\section{Future topics for RSV research}

Intensive research has been conducted on RSV for the past several decades. Research topics have included: determination of RSV pathogenicity, replication, and movement; identification of rice varieties or loci resistant to RSV; mechanisms of infection and resistance to infection in rice; and interactions among RSV-rice-SBPH. Compared to what is known about other plant viruses, however, relatively little is known about RSV.

To prevent or reduce diseases caused by RSV, research should characterize the functions of all RSV proteins. Although several QTL analyses narrowed down the location of RSV resistance genes to chromosome 11, rice genes conferring resistance to RSV have not been identified. RSV resistance genes should be identified soon, however, because of ongoing research on QTL analysis and fine mapping. Molecular mechanisms underlying RSV infection and resistance remain unclear. To generate RSV-resistant rice cultivars, breeders will need to follow multiple approaches. First, QTLs in different cultivars or varieties should be evaluated based on several parameters including infection rates, disease rating index, and the ranking of the RSV resistance in the greenhouse and field. Moreover, maintenance of RSV varies among SBPH biotypes, and this difference among biotypes should be further characterized and explained. Because $S t v b-i$ has been used to confer RSV resistance in japonica cultivars for 40 years, it is highly possible that $S t v b$ - $i$-mediated RSV resistance could breakdown in the near future. Therefore, researchers should consider all possible QTLs for molecular breeding as well as the genetic modification of rice plants.

Almost nothing is known about the RSV-host interaction. Although three rice proteins that interact with RSV 
proteins have been identified, their functions have not been characterized. Our research group is now conducting next generation sequencing to examine the rice transcriptome at different times after RSV infection. We will identify differentially expressed mRNAs and microRNAs as well as siRNAs derived from RSV. Combined data sets will provide insights into the RSV-host interaction at the transcriptional level. Because RSV is transmitted by SBPH, the interactions among RSV, rice, and SBPH should also be studied.

Given the complexity of the biology, a better understanding of the interactions among RSV, SBPH, and rice will require the integration of several approaches and collaboration among plant pathologists, molecular biologists, and entomologists.

\section{Acknowledgements}

This research was supported in part by grants from the Next-Generation BioGreen 21 Program (No. PJ00819801), Rural Development Administration, the National Research Foundation grant (No. 20110012328) funded by the Ministry of Education, Science, and Technology (MEST), and the Vegetable Breeding Research Center (No. 71000103) through Agriculture Research Center program from the Ministry for Food, Agriculture, Forestry and Fisheries, Republic of Korea. WKC, SL, and SHP were supported by research fellowships from the Brain Korea 21 Project.

\section{References}

Abo, M. E. and Sy, A. A. 1997. Rice virus diseases: epidemiology and management strategies. J. Sustain. Agr. 11:113-134.

Barbier, P., Takahashi, M., Nakamura, I., Toriyama, S. and Ishihama, A. 1992. Solubilization and promoter analysis of RNA polymerase from Rice stripe virus. J. Virol. 66:6171-6174.

Cai, L., Ma, X., Lin, K., Deng, K., Zhao, S. and Li, C. 2003. Detecting Rice stripe virus (RSV) in the small brown planthopper Laodelphax striatellus with high specificity by RT-PCR. J. Virol. Methods 112:115-120.

Cheng, E. and Mir, M. A. 2012. Signatures of host mRNA 5' terminus for efficient Hantavirus cap snatching. J. Virol. 86: 10173-10185.

Dias, A., Bouvier, D., Crépin, T., McCarthy, A. A., Hart, D. J., Baudin, F., Cusack, S. and Ruigrok, R. W. H. 2009. The capsnatching endonuclease of influenza virus polymerase resides in the PA subunit. Nature 458:914-918.

Ding, X. L., Jiang, L., Wang, C. M., CHen, L. M., Cheng, Z. B., Fan, Y. J., Zhou, Y. J. and Wan, J. M. 2004. QTL analysis for rice stripe disease resistance gene using recombinant inbred lines (RILs) derived from crossing of Kinmaze and DV85. $J$. Gen. Genom. 31:287-292.

Du, P., Wu, J., Zhang, J., Zhao, S., Zheng, H., Gao, G., Wei, L. and
Li, Y. 2011. Viral infection induces expression of novel phased microRNAs from conserved cellular microRNA precursors. PLoS Pathog. 7:e1002176.

Du, Z., Xiao, D., Wu, J., Jia, D., Yuan, Z., Liu, Y., Hu, L., Han, Z., Wei, T., Lin, Q., Wu, Z. and Xie, L. 2011. p2 of Rice stripe virus (RSV) interacts with OsSGS3 and is a silencing suppressor. Mol. Plant Pathol. 12:808-814.

Falk, B. W. and Tsai, J. H. 1998. Biology and molecular biology of viruses in the genus Tenuivirus. Annu. Rev. Phytopathol. 36:139-163.

Hamamatsu, C., Toriyama, S., Toyoda, T. and Ishihama, A. 1993. Ambisense coding strategy of the Rice stripe virus genome: in vitro translation studies. J. Gen. Virol. 74:1125.

Hao, Z., Wang, L., He, Y., Liang, J. and Tao, R. 2011. Expression of defense genes and activities of antioxidant enzymes in rice resistance to Rice stripe virus and small brown planthopper. Plant Physiol. Biochem. 49:744-751.

Hayakawa, T., Zhu, Y., Itoh, K., Kimura, Y., Izawa, T., Shimamoto, K. and Toriyama, S. 1992. Genetically engineered rice resistant to Rice stripe virus, an insect-transmitted virus. Proc. Natl. Acad. Sci. 89:9865.

Hayano-Saito, Y., Saito, K., Nakamura, S., Kawasaki, S. and Iwasaki, M. 2000. Fine physical mapping of the rice stripe resistance gene locus, Stvb-i. Theor. Appl. Genet. 101:59-63.

Hayano-Saito, Y., Tsuji, T., Fujii, K., Saito, K., Iwasaki, M. and Saito, A. 1998. Localization of the rice stripe disease resistance gene, Stv-bi, by graphical genotyping and linkage analyses with molecular markers. Theor. Appl. Genet. 96:10441049.

Hayano, Y., Kakutani, T., Hayashi, T. and Minobe, Y. 1990. Coding strategy of Rice stripe virus: major nonstructural protein is encoded in viral RNA segment 4 and coat protein in RNA complementary to segment 3. Virology 177:372-374.

Hibino, H. 1996. Biology and epidemiology of rice viruses. Annu. Rev. Phytopathol. 34:249-274.

Ise, K., Ishikawa, K., Li, C. and Ye, C. 2002. Inheritance of resistance to Rice stripe virus in rice line 'BL 1'. Euphytica 127:185-191.

Ishikawa, K., Omura, T. and Hibino, H. 1989. Morphological characteristics of Rice stripe virus. J. Gen. Virol. 70:34653468.

Jiang, L., Qian, D., Zheng, H., Meng, L. Y., Chen, J., Le, W. J., Zhou, T., Zhou, Y. J., Wei, C. H. and Li, Y. 2012. RNA-dependent RNA polymerase 6 of rice (Oryza sativa) plays role in host defense against negative-strand RNA virus, Rice stripe virus. Virus Res. 163:512-519.

Jonson, M. G., Choi, H. S., Kim, J. S., Choi, I. R. and Kim, K. H. 2009. Complete genome sequence of the RNAs 3 and 4 segments of Rice stripe virus isolates in Korea and their phylogenetic relationships with Japan and China isolates. Plant Pathol. J. 25:142-150.

Jonson, M. G., Choi, H. S., Kim, J. S., Choi, I. R. and Kim, K. H. 2009. Sequence and phylogenetic analysis of the RNA1 and RNA2 segments of Korean Rice stripe virus isolates and comparison with those of China and Japan. Arch. Virol. 154:17051708 . 
Kakutani, T., Hayano, Y., Hayashi, T. and Minobe, Y. 1990. Ambisense segment 4 of Rice stripe virus: possible evolutionary relationship with phleboviruses and uukuviruses (Bunyaviridae). J. Gen Virol. 71:1427-1432.

Kakutani, T., Hayano, Y., Hayashi, T. and Minobe, Y. 1991. Ambisense segment 3 of Rice stripe virus: the first instance of a virus containing two ambisense segments. J. Gen. Virol. 72:465-468.

Kim, K., Choi, D., Kim, S. M., Kwak, D. Y., Choi, J., Lee, S., Lee, B. C., Hwang, D. and Hwang, I. 2012. A systems approach for identifying resistance factors to Rice stripe virus. Mol. Plant Microbe. Interact. 25:534-545.

Kisimoto, R. 1967. Genetic variation in the ability of a planthopper vector; Laodelphax striatellus (Fallén) to acquire the Rice stripe virus. Virology 32:144-152.

Kwon, T., Lee, J. H., Park, S. K., Hwang, U. H., Cho, J. H., Kwak, D. Y., Youn, Y. N., Yeo, U. S., Song, Y. C. and Nam, J. 2012. Fine mapping and identification of candidate rice genes associated with $q S T V 11^{S G}$, a major QTL for rice stripe disease resistance. Theor. Appl. Genet. 125:1-14.

Le, D. T., Netsu, O., Uehara-Ichiki, T., Shimizu, T., Choi, I. R., Omura, T. and Sasaya, T. 2010. Molecular detection of nine rice viruses by a reverse-transcription loop-mediated isothermal amplification assay. J. Virol. Methods 170:90-93.

Lee, B. C., Yoon, Y. N., Hong, S. J., Hong, Y. K., Kwak, D. Y., Lee, J. H., Yae, U. S., Kang, H. W. and Hwang, H. G. 2008. Analysis on the occurrence of Rice stripe virus. Res. Plant Dis. 14:210-213.

Li, S., Li, X., Sun, L. and Zhou, Y. 2012. Analysis of Rice stripe virus whole-gene expression in rice and in the small brown planthopper by real-time quantitative PCR. Acta Virol. 56:7579.

Li, S., Xiong, R., Wang, X. and Zhou, Y. 2011. Five proteins of Laodelphax striatellus are potentially involved in the interactions between Rice stripe virus and vector. PLoS One $6: \mathrm{e} 26585$.

Lian, S., Jonson, M. G., Cho, W. K., Choi, H. S., Je, Y. H. and Kim, K. H. 2011. Generation of antibodies against Rice stripe virus proteins based on recombinant proteins and synthetic polypeptides. Plant Pathol. J. 27:37-43.

Liang, D., Ma, X., Qu, Z. and Hull, R. 2005. Nucleic acid binding property of the gene products of Rice stripe virus. Virus Genes 31:203-209.

Liang, D., Qu, Z., Ma, X. and Hull, R. 2005. Detection and localization of Rice stripe virus gene products in vivo. Virus Genes 31:211-221.

Lu, L., Du, Z., Qin, M., Wang, P., Lan, H., Niu, X., Jia, D., Xie, L. and Lin, Q. 2009. Pc4, a putative movement protein of Rice stripe virus, interacts with a type I DnaJ protein and a small Hsp of rice. Virus Genes 38:320-327.

Ma, J., Song, Y., Wu, B., Jiang, M., Li, K., Zhu, C. and Wen, F. 2011. Production of transgenic rice new germplasm with strong resistance against two isolations of Rice stripe virus by RNA interference. Transgenic Res. 20:1367-1377.

Maeda, H., Matsushita, K., Iida, S. and Sunohara, Y. 2006. Characterization of two QTLs controlling resistance to Rice stripe virus detected in a Japanese upland rice line, Kanto 72. Breeding Sci. 56:359-364.

McCough, S. R. and Doerge, R. W. 1995. QTL mapping in rice. Trends Genet. 11:482-487.

Notomi, T., Okayama, H., Masubuchi, H., Yonekawa, T., Watanabe, K., Amino, N. and Hase, T. 2000. Loop-mediated isothermal amplification of DNA. Nucleic Acids Res. 28:e63-e63.

Nuruzzaman, M., Manimekalai, R., Sharoni, A. M., Satoh, K., Kondoh, H., Ooka, H. and Kikuchi, S. 2010. Genome-wide analysis of NAC transcription factor family in rice. Gene 465:30-44.

Otuka, A., Matsumura, M., Sanada-Morimura, S., Takeuchi, H., Watanabe, T., Ohtsu, R. and Inoue, H. 2010. The 2008 overseas mass migration of the small brown planthopper, Laodelphax striatellus, and subsequent outbreak of rice stripe disease in western Japan. Appl. Entomol. Zoo. 45:259-266.

Pan, X., Liang, G., Chen, Z. and Zhang, Y. 2005. Breeding strategy on resistance to rice stripe in Jiangsu. Jiangsu Agr. Sci. 5:22-23.

Pan, X. B., Chen, Z. X., Zuo, S. M., Zhang, Y. F., Wu, X. J., Ma, N., Jiang, Q. X., Que, J. H. and Zhou, C. H. 2009. A new rice cultivar Wulingjing 1 resistant to Rice stripe virus developed by marker assisted selection. Acta Agron. Sin. 35:1851-1857.

Park, H. M., Choi, M. S., Kwak, D. Y., Lee, B. C., Lee, J. H., Kim, M. K., Kim, Y. G., Shin, D. B., Park, S. K. and Kim, Y. H. 2012. Suppression of NS3 and MP is important for the stable inheritance of RNAi-mediated Rice stripe virus (RSV) resistance obtained by targeting the fully complementary RSV-CP gene. Mol. Cells 33:1-9.

Satoh, K., Kondoh, H., Sasaya, T., Shimizu, T., Choi, I. R., Omura, T. and Kikuchi, S. 2010. Selective modification of rice (Oryza sativa) gene expression by Rice stripe virus infection. J. Gen. Virol. 91:294-305.

Sharoni, A. M., Nuruzzaman, M., Satoh, K., Shimizu, T., Kondoh, H., Sasaya, T., Choi, I. R., Omura, T. and Kikuchi, S. 2011. Gene structures, classification and expression models of the AP2/EREBP transcription factor family in rice. Plant Cell Physiol. 52:344-360.

Shen, M., Xu, Y., Jia, R., Zhou, X. and Ye, K. 2010. Size-independent and noncooperative recognition of dsRNA by the Rice stripe virus RNA silencing suppressor NS3. J. Mol. Biol. 404:665.

Shimizu, T., Nakazono-Nagaoka, E., Uehara-Ichiki, T., Sasaya, T. and Omura, T. 2011. Targeting specific genes for RNA interference is crucial to the development of strong resistance to Rice stripe virus. Plant Biotechnol. J. 9:503-512.

Shimizu, T., Toriyama, S., Takahashi, M., Akutsu, K. and Yoneyama, K. 1996. Non-viral sequences at the 5 termini of mRNAs derived from virus-sense and virus-complementary sequences of the ambisense RNA segments of Rice stripe tenuivirus. J. Gen. Virol. 77:541-546.

Sun, F., Yuan, X., Zhou, T., Fan, Y. and Zhou, Y. 2011. Arabidopsis is Susceptible to Rice stripe virus Infections. J. Phytopathol. 159:767-772.

Suzuki, Y., Fuji, S., Takahashi, Y. and Kojima, M. 1992. Immunogold localization of Rice stripe virus particle antigen in thin 
sections of insect host cells. Ann. Phytopathol. Soc. Jpn. 58:480-480.

Takahashi, M., Goto, C., Ishikawa, K., Matsuda, I., Toriyama, S. and Tsuchiya, K. 2003. Rice stripe virus $23.9 \mathrm{~K}$ protein aggregates and forms inclusion bodies in cultured insect cells and virus-infected plant cells. Arch. Virol. 148:2167-2179.

Takahashi, M., Toriyama, S., Hamamatsu, C. and Ishihama, A. 1993. Nucleotide sequence and possible ambisense coding strategy of Rice stripe virus RNA segment 2. J. Gen. Virol. 74:769-773.

Takahashi, M., Toriyama, S., Kikuchi, Y., Hayakawa, T. and Ishihama, A. 1990. Complementarity between the 5'-and 3'-terminal sequences of Rice stripe virus RNAs. J. Gen. Virol. 71:2817-2821.

Takahashi, Y., Omura, T., Shohara, K. and Tsuchizaki, T. 1991. Comparison of four serological methods for practical detection of ten viruses of rice in plants and insects. Plant Dis. 75:458-461.

Toriyama, S., Takahashi, M., Sano, Y., Shimizu, T. and Ishihama, A. 1994. Nucleotide sequence of RNA 1, the largest genomic segment of Rice stripe virus, the prototype of the tenuiviruses. J. Gen. Virol. 75:3569.

Wang, B., Jiang, L., Zhang, Y., Zhang, W., Wang, Q., Liu, S., Liu, Y., Cheng, X., Zhai, H. and Wan, J. 2011. Genetic dissection of the resistance to Rice stripe virus present in the indica rice cultivar 'IR24'. Genome 54:611-619.

Wang, H. D., Chen, J. P., Zhang, H. M., Sun, X. L., Zhu, J. L., Wang, A. G., Sheng, W. X. and Adams, M. 2008. Recent Rice stripe virus epidemics in Zhejiang province, China, and experiments on sowing date, disease-yield loss relationships, and seedling susceptibility. Plant Dis. 92:1190-1196.

Wang, Y., Xue, Y. and Li, J. 2005. Towards molecular breeding and improvement of rice in China. Trends Plant Sci. 10:610614.

Washio, O., Ezuka, A., Toriyama, K. and Sakurai, Y. 1968. Studies on the breeding of rice varieties resistant to stripe disease II. Genetic study on resistance to stripe disease in Japanese upland rice. Jpn. J. Breed 18:96-101.

Washio, O., Ezuka, A., Toriyama, K. and Sakurai, Y. 1968. Studies on the breeding of rice varieties resistant to stripe disease III. Genetic studies on resistance to stripe disease in foreign varieties. Jpn. J. Breed 18:167-172.

Wei, T. Y., Yang, J. G., Liao, F. L., Gao, F. L., Lu, L. M., Zhang, X. T., Li, F., Wu, Z. J., Lin, Q. Y. and Xie, L. H. 2009. Genetic diversity and population structure of Rice stripe virus in China. J. Gen. Virol. 90:1025.

Wu, S. J., Zhong, H., Zhou, Y., Zuo, H., Zhou, L. H., Zhu, J. Y., Ji, C. Q., Gu, S. L., Gu, M. H. and Liang, G. H. 2009. Identification of QTLs for the resistance to Rice stripe virus in the indica rice variety Dular. Euphytica 165:557-565.

Wu, X., Zuo, S., Chen, Z., Zhang, Y., Zhu, J., Ma, N., Tang, J., Chu, C. and Pan, X. 2011. Fine mapping of $q S T V 11^{T Q}$, a major gene conferring resistance to rice stripe disease. Theor. Appl. Genet. 122:915-923.

Xiong, R., Wu, J., Zhou, Y. and Zhou, X. 2008. Identification of a movement protein of the tenuivirus Rice stripe virus. J. Virol.
82:12304-12311.

Xiong, R., Wu, J., Zhou, Y. and Zhou, X. 2009. Characterization and subcellular localization of an RNA silencing suppressor encoded by Rice stripe tenuivirus. Virology 387:29-40.

Yan, F., Zhang, H., Adams, M. J., Yang, J., Peng, J., Antoniw, J. F., Zhou, Y. and Chen, J. 2010. Characterization of siRNAs derived from Rice stripe virus in infected rice plants by deep sequencing. Arch. Virol. 155:935-940.

Yang, J. G., Wang, W. T., Ding, X. L., Guo, L. J., Fang, Z. X., Xie, L. Y., Lin, Q. Y., Wu, Z. J. and Xie, L. H. 2010. Auxin regulation in the interaction between Rice stripe virus and rice. Chinese J. Agr. Biotechnol. 6:27.

Yao, M., Zhang, T., Zhou, T., Zhou, Y., Zhou, X. and Tao, X. 2012. Repetitive prime-and-realign mechanism converts short capped RNA leaders into longer ones that may be more suitable for elongation during Rice stripe virus transcription initiation. J. Gen. Virol. 93:194-202.

Yuan, Z., Chen, H., Chen, Q., Omura, T., Xie, L., Wu, Z. and Wei, T. 2011. The early secretory pathway and an actin-myosin VIII motility system are required for plasmodesmatal localization of the NSvc4 protein of Rice stripe virus. Virus Res. 159:62-68.

Zhang, C., Pei, X., Wang, Z., Jia, S., Guo, S., Zhang, Y. and Li, W. 2012. The Rice stripe virus pc4 functions in movement and foliar necrosis expression in Nicotiana benthamiana. Virology 425:113-121.

Zhang, C., Song, Y., Jiang, F., Li, G., Jiang, Y., Zhu, C. and Wen, F. 2012. Virus resistance obtained in transgenic tobacco and rice by RNA interference using promoters with distinct activity. Biol. Plantarum 56:742-748.

Zhang, H. M., Yang, J., Sun, H. R., Xin, X., Wang, H. D., Chen, J. P. and Adams, M. 2007. Genomic analysis of Rice stripe virus Zhejiang isolate shows the presence of an OTU-like domain in the RNA1 protein and a novel sequence motif conserved within the intergenic regions of ambisense segments of tenuiviruses. Arch. Virol. 152:1917-1923.

Zhang, S., Li, L., Wang, X. and Zhou, G. 2007. Transmission of Rice stripe virus acquired from frozen infected leaves by the small brown planthopper (Laodelphax striatellus Fallen). J. Virol. Methods 146:359-362.

Zhang, X., Wang, X. and Zhou, G. 2008. A one-step real time RTPCR assay for quantifying Rice stripe virus in rice and in the small brown planthopper (Laodelphax striatellus Fallen). J. Virol. Methods 151:181-187.

Zhang, Y. X., Wang, Q., Jiang, L., Liu, L. L., Wang, B. X., Shen, Y. Y., Cheng, X. N. and Wan, J. 2011. Fine mapping of $q S T V 11^{K A S}$, a major QTL for rice stripe disease resistance. Theor. Appl. Gen. 122:1591-1604.

Zhang, Y. X., Wang, Q., Jiang, L., Wang, B. X., Liu, L. L., Shen, Y. Y., Cheng, X. N. and Wan, J. M. 2012. Detection and fine mapping of two quantitative trait loci for partial resistance to stripe virus in rice (Oryza sativa L.). Mol. Breeding 30:1-13.

Zhao, F., Cai, Z., Hu, T., Yao, H., Wang, L., Dong, N., Wang, B., $\mathrm{Ru}, \mathrm{Z}$. and Zhai, W. 2010. Genetic analysis and molecular mapping of a novel gene conferring resistance to Rice stripe virus. Plant Mol. Biol. Rep. 28:512-518. 
Zhao, S., Dai, X., Liang, J. and Liang, C. 2012. Surface display of Rice stripe virus $\mathrm{NSvc} 2$ and analysis of its membrane fusion activity. Virol. Sin. 27:100-108.

Zhao, S., Zhang, G., Dai, X., Hou, Y., Li, M., Liang, J. and Liang, C. 2012. Processing and intracellular localization of Rice stripe virus Pc2 protein in insect cells. Virology 429:148-154.

Zhou, Y., Yuan, Y., Yuan, F., Wang, M., Zhong, H., Gu, M. and Liang, G. 2012. RNAi-directed down-regulation of RSV results in increased resistance in rice (Oryza sativa L.). Bio- technol. Lett. 34:965-972.

Zhu, Y., Hayakawa, T. and Toriyama, S. 1992. Complete nucleotide sequence of RNA 4 of Rice stripe virus isolate T, and comparison with another isolate and with Maize stripe virus. J. Gen. Virol. 73:1309-1312.

Zhu, Y., Hayakawa, T., Toriyama, S. and Takahashi, M. 1991. Complete nucleotide sequence of RNA 3 of Rice stripe virus: an ambisense coding strategy. J. Gen. Virol. 72:763. 